\title{
La Maison de la Recherche et de l'Imagination à Caen : un tiers lieu pour la recherche et l'innovation ouverte
}

Bruno Dosseur

\section{OpenEdition}

Journals

Édition électronique

URL : http://journals.openedition.org/ocim/1557

DOI : $10.4000 /$ ocim. 1557

ISSN : 2108-646X

Éditeur

OCIM

Édition imprimée

Date de publication : 1 septembre 2015

Pagination : 5-11

ISSN : 0994-1908

\section{Référence électronique}

Bruno Dosseur, « La Maison de la Recherche et de I'Imagination à Caen : un tiers lieu pour la recherche et l'innovation ouverte ", La Lettre de I'OCIM [En ligne], 161 | 2015, mis en ligne le 01 septembre 2016, consulté le 05 mai 2019. URL : http://journals.openedition.org/ocim/1557 ; DOI : 10.4000/ocim.1557

Ce document a été généré automatiquement le 5 mai 2019.

Tous droits réservés 


\section{La Maison de la Recherche et de l'Imagination à Caen : un tiers lieu pour la recherche et l'innovation ouverte}

Bruno Dosseur

La Maison de la Recherche et de l'Imagination à Caen : un tiers lieu pour la recherche et l'innovation ouverte

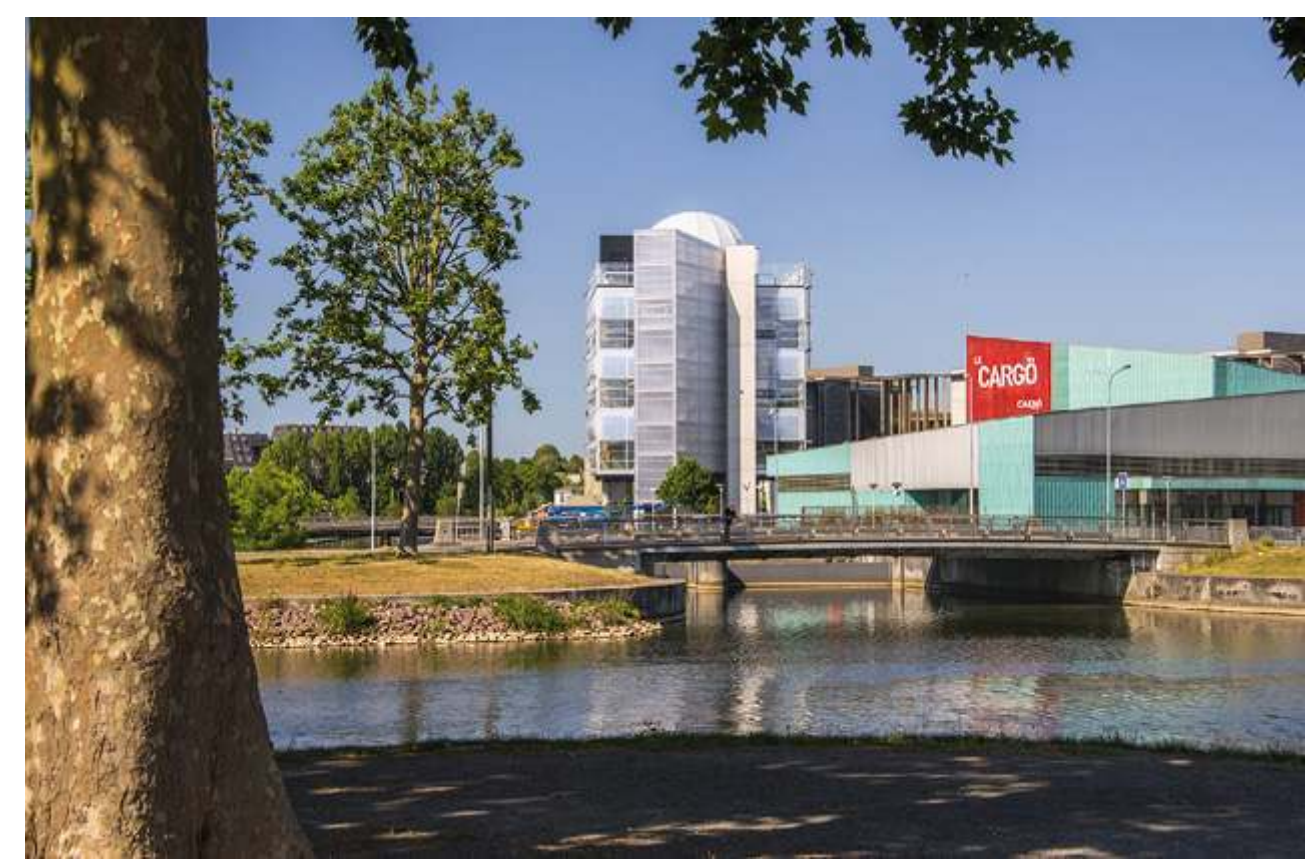

(C) Relais d'sciences/G. Dupuy

En moins de huit mois, une tour de 34 mètres de haut, faisant vaguement penser au robot R2-D2, s'est élevée au cœur de Caen dans un ancien espace industriel en plein 
renouvellement urbain. Elle porte le nom de code, encore provisoire, de Maison de la Recherche et de l'Imagination (MRI) et s'intègre à un ensemble culturel très riche : une bibliothèque signée Rem Koolhaas (BMVR) livrée en 2016, une salle de musiques actuelles (Le Cargö), l'École Supérieure des Arts et Media (ESAM Caen-Cherbourg), un espace de création et de diffusion artistique (La Fermeture Éclair) et un espace d'interprétation de l'urbanisme et de l'architecture (Le Pavillon).

Ce bâtiment, imaginé et construit par Relais d'sciences, CCSTI de Basse-Normandie, est l'une des réalisations les plus radicales d'Inmediats, projet d'investissement d'avenir porté par six centres de sciences ${ }^{1}$. Achevé en juin 2015, il ouvre progressivement ses portes aux publics pendant le dernier trimestre 2015. Inmediats s'est donné pour objectif de refonder le rôle et la place des CCSTI dans trois dimensions : proposer un nouveau projet culturel basé sur le FAIRE et prenant en compte, tout particulièrement, les 15-25 ans; construire des espaces adaptés aux nouvelles formes de médiation nées avec le numérique; développer collectivement un nouveau modèle économique prenant en compte la raréfaction des fonds publics.

3 Pour Relais d'sciences et ses partenaires, un centre de sciences de nouvelle génération est un lieu qui éclaire l'avenir dans sa dimension recherche et technologies en intégrant les nouvelles méthodes d'organisation collective et de partage de l'innovation : recherche et innovation responsables $\left(\mathrm{RRI}^{2}\right)$, intelligence collective et co-construction des savoirs, innovation ouverte et design thinking, économie du partage et innovation sociale, do it with others et open source.

4 La MRI s'inscrit clairement dans ces tentatives de construction d'une nouvelle forme de partage de la connaissance et de culture de l'innovation, qu'elle soit technologique ou non. Ce parti pris va jusqu'à refonder la gouvernance même d'un centre culturel pour garantir sa capacité de création collective et la mobilisation durable de ses partenaires et de ses publics.

5 Les sources d'inspiration de la MRI dépassent le champ traditionnel de la culture scientifique. Les autres établissements d'Inmediats sont les premiers modèles de référence par leur expérience et la maîtrise de développement tous très différents. Mais la MRI s'inspire également librement des dynamiques à l'œuvre au sein de la Gaité Lyrique, du 104, de la Ruche, de Numa, de la Science Gallery à Dublin, de Paris\&Co Incubateurs, du Point $\mathrm{H}^{\wedge} \mathrm{HT}$ à Saint-Pierre-des-Corps, de l'île de la création à Nantes, du Centre des arts d'Enghien-les-Bains, d'Erasme à Lyon et de beaucoup d'autres. 
À l'image des Parcours culturels développés par Relais d'sciences, Inmédiats propose un nouveau projet culturel basé sur le FAIRE et prenant en compte, tout particulièrement, les jeunes adultes.

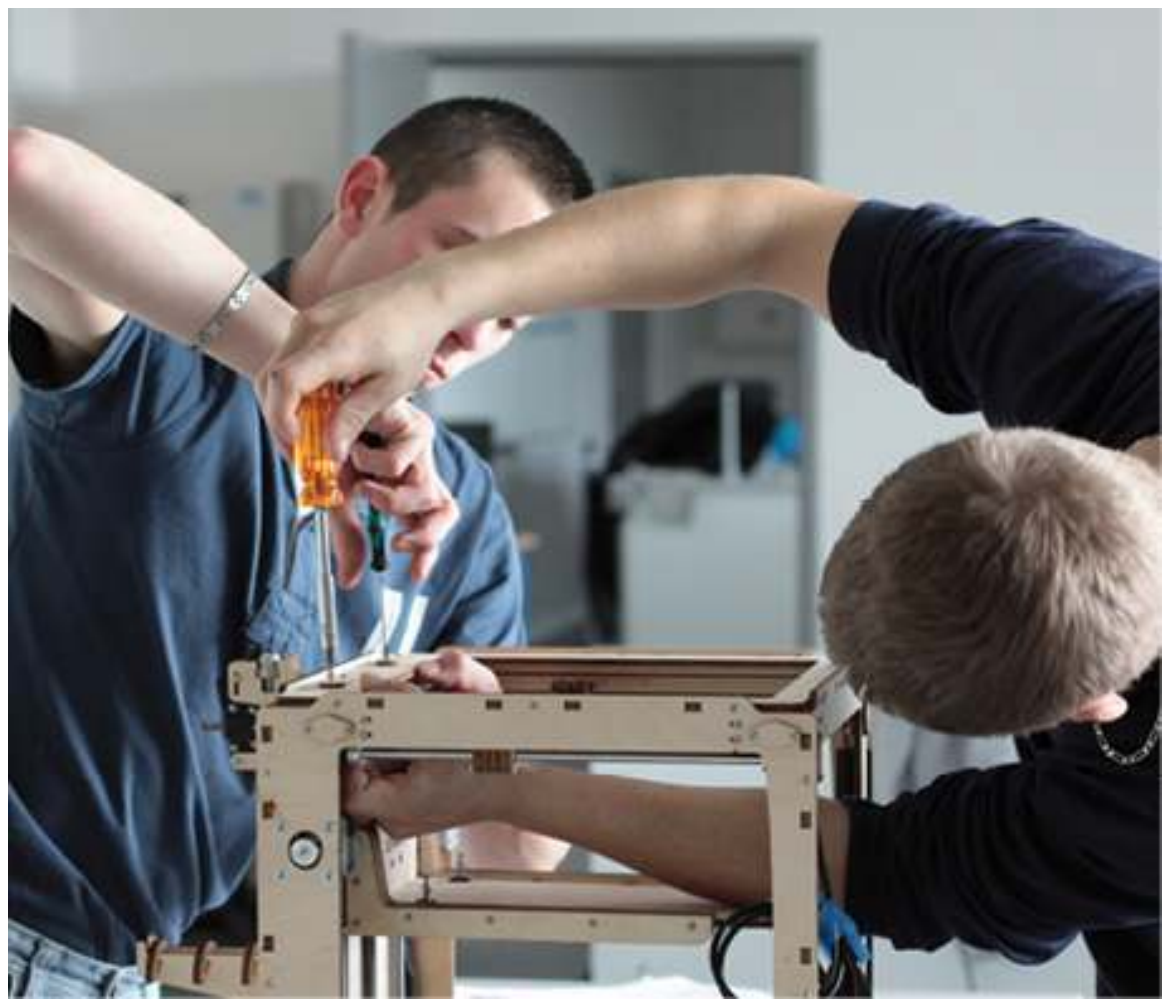

(C) CFA-BTP ORNE/M. TABURET

\section{Le projet culturel}

Il est nécessaire d'insister ici sur le fait que c'est bien le projet culturel qui a créé le bâtiment et non l'inverse. À l'heure où tant de bâtiments industriels en reconversion cherchent désespérément un projet pour les habiter, chaque parcelle de la MRI est la résultante d'une démarche volontaire. La MRI est donc un outil au service d'un projet et non le projet lui-même.

7 La définition du lieu, sa bonne réalisation en terme d'usages professionnels et publics, son implantation dans le réseau de partenaires, le choix de ses équipements ont été conduits par l'équipe de Relais d'sciences, notamment au sein de l'équipe de direction (Matthieu Debar, Bruno Dosseur et François Millet, rejoints depuis octobre 2014 par le régisseur général de la MRI, Gilles Hardoin). Guillaume Dupuy a développé des outils originaux pour une narration des projets et réalisations sur www.relais-sciences.org. Virginie Klauser, Stéphane Lebrun, Julien Lefebvre et Christine Chiamone ont apporté leurs compétences techniques, d'animation et de suivi administratif pour consolider le projet et les actions en cours.

8 Le projet culturel de la MRI est fondé sur celui d'Inmediats. Relais d'sciences en a fait le cœur de sa stratégie. Cette démarche impose de ne pas transiger avec cinq principes et objectifs d'Inmediats devenus les clés de voûte de la MRI :

9 - impliquer le public dans la démarche d'innovation et de recherche ;

10 - s'adresser prioritairement aux jeunes adultes ; 
11 système français à s'adapter aux crises économiques en faisant un parallèle avec la théorie de l'évolution 4 . Il rappelle que l'adaptation darwinienne qui fait la force de la nature est fondée sur la diversification et la sélection. Par analogie, tout système de société désireux de surmonter les inévitables crises, doit favoriser la singularité, l'originalité, la diversité des sources d'innovation et de créativité puis sélectionner les idées et projets les plus aptes à s'adapter à la situation présente. Ce modèle doit être perpétuellement en mouvement et ne jamais considérer comme définitivement acquis un mode de production, une filière, une spécialité. susceptible d'innover en matière de culture scientifique afin de contribuer davantage au dynamisme des territoires. Ainsi, la MRI se veut un laboratoire de la médiation culturelle autour de la recherche et de l'innovation. La participation du public y est recherchée en permanence, non pas simplement à travers des procédés interactifs mais bien dans l'émergence de nouvelles idées et de nouveaux usages portés par les utilisateurs (Living $\mathrm{Lab}$ ), la production de prototypes via des machines à commande numérique (Fab Lab), la participation à des programmes de recherche ou de prospective territoriale (Living City, tourisme numérique, robotique humanoïde).

17 Pour y parvenir, une vingtaine de structures partenaires sont intégrées physiquement dans le bâtiment, à titre permanent ou temporaire: enseignants-chercheurs ou doctorants, membres de start-ups, de réseaux consulaires ou d'agence d'innovation, designers, membres d'association, de collectivités ou d'organismes culturels, artistes... L'innovation porte donc autant sur l'aménagement et l'occupation collective des locaux que sur l'organisation administrative, la création d'espaces interstitiels pour le public et les professionnels, la programmation culturelle, la relation au territoire...

\section{S'adresser prioritairement aux jeunes adultes}

18 La mobilisation des 15-25 ans sur les lieux culturels est une gageure si une démarche volontariste n'est pas réellement engagée. Elle dépasse les simples problèmes de communication et de gratuité et impose la définition d'une véritable stratégie qui repose sur cinq axes :

19 - une place dévolue aux 15-25 ans: le principe d'Inmediats est de rompre avec le positionnement habituel des jeunes dans les centres de science dont la participation se limite souvent à une activité en groupe classe très encadrée et basée sur la transmission des savoirs. Fidèle à sa vocation de co-construction des savoirs, la MRI doit valoriser leur 
expression et mettre ces jeunes en situation de concevoir et réaliser par eux-mêmes. Elle s'adresse à eux en qualité d'adultes, capables d'apporter leur contribution à des projets dont certains à vocation professionnelle ;

20 - un bâtiment appropriable : son identité et sa conception même intègrent l'objectif d'en faire un " quartier général » pour les jeunes. Il doit s'adresser clairement à eux, leur offrir des espaces pouvant être investis en petits groupes, leur permettre d'y travailler en mode nomade, d'y présenter leurs projets, contributions et réalisations, être d'un accès spontané et facile, convivial, accessible financièrement, original et jouable, repéré dans les réseaux sociaux. L'implantation de la MRI au cœur de la vie culturelle caennaise permet d'imaginer une forte complémentarité des publics. L'absence de billetterie, si elle ne signifie pas la gratuité de toutes les opérations proposées, doit faciliter l'appropriation du site et un retour très régulier des usagers;

21 - des formats attractifs et adaptés à leurs modes de vie : rencontres privilégiées avec de jeunes inventeurs, entrepreneurs et chercheurs, activités en soirées et nocturnes, fêtes, avant-premières technologiques... Tous les formats incitent, après une découverte éventuelle lors d'une visite par groupes captifs (lycéens, apprentis par exemple) à s'impliquer progressivement dans une démarche volontaire hors temps scolaire ;

22 - une identité qui leur correspond : le nom même de l'établissement (encore à l'étude), sa charte graphique, les valeurs qu'il défend, son intégration dans les réseaux sociaux, sont autant de marqueurs à prendre en compte très sérieusement. Il en est de même sur les manifestations proposées et leur mode de diffusion. Ils doivent être particulièrement cohérents avec la promesse de l'établissement ;

23 - des partenariats axés prioritairement sur ces publics: intégration dans les festivals musicaux et artistiques adaptés, partenariat avec de grandes enseignes de technologies, avec les associations étudiantes et lycéennes, les radios, intégration dans les communautés virtuelles, mobilisation des écoles et parcours de formation intégrant la créativité, propositions d'acquisition de compétences en mode informel, intégration des dispositifs sur l'entreprenariat des jeunes... 
Tout comme Jean-Marc Routoure, enseignant-chercheur, une vingtaine de structures partenaires seront intégrées dans le bâtiment.

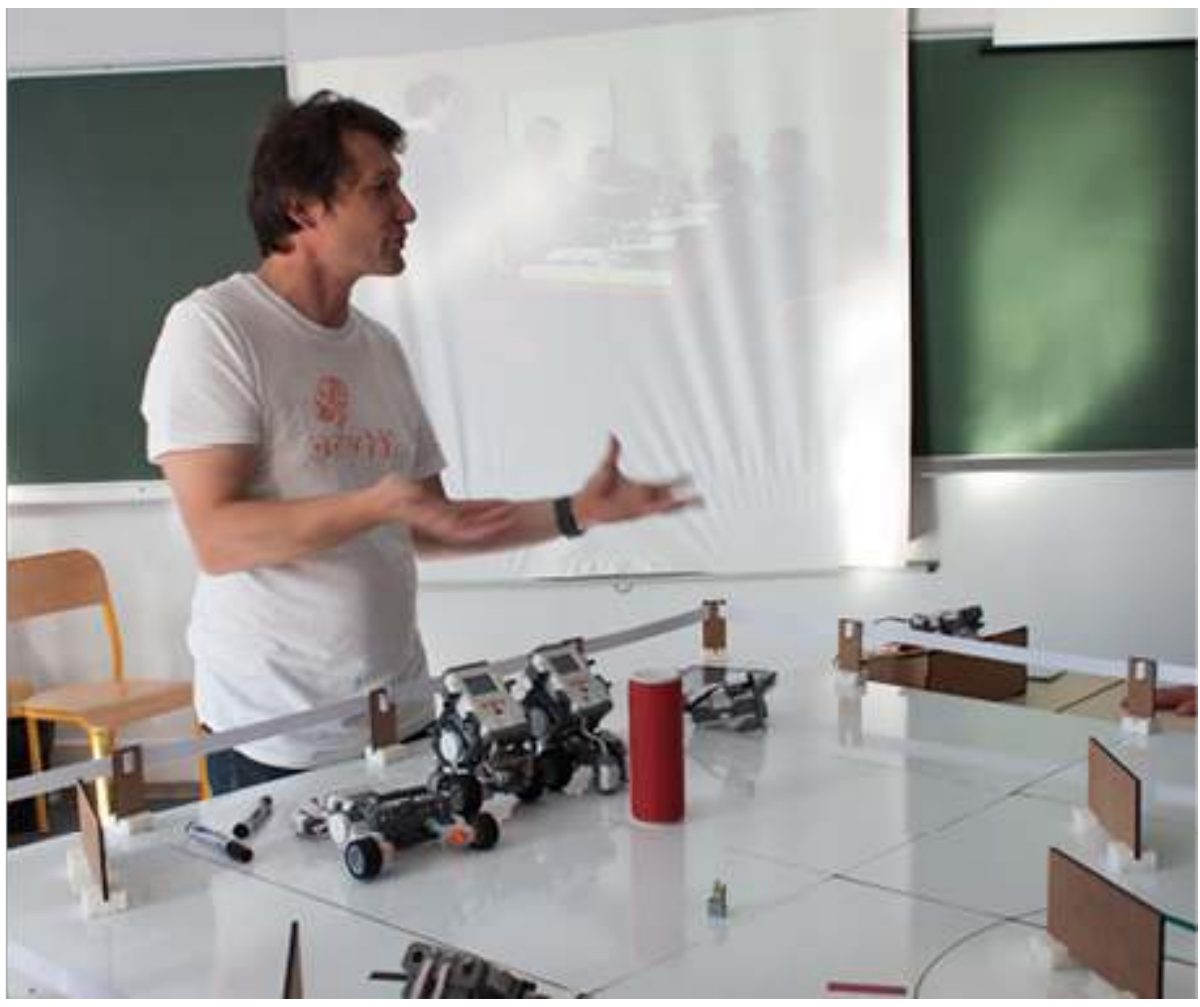

(c) Université de Caen Normandie

\section{Assurer la mixité des publics professionnels et particuliers}

La MRI est conçue comme un lieu de convivialité, de découverte et de travail destiné prioritairement à des adultes. Elle accueille des formats d'évènements très variés ; des activités culturelles aux séminaires professionnels sur la créativité, le design et l'innovation en passant par des workshops sur les objets connectés ou des installations artistiques présentées dans le cadre d'un festival.

Le principe de programmation de la MRI repose sur la synchronisation de ces évènements, tous en lien avec la recherche, l'innovation ou la culture technique et industrielle, proposés à des communautés d'intérêts différentes. Dès lors, le croisement entre les différents publics est facilité, voire provoqué. Il est ainsi proposé aux organismes qui réservent la MRI pour organiser leurs séminaires professionnels de susciter des temps de rencontre avec le public: intégration d'ateliers créatifs, d'expérimentations ou de tests d'usages autour d'une innovation (Living Lab), conférence, démonstrations de prototypes, master class... Sauf cas exceptionnel, le Fab Lab et le bar ne sont pas privatisés afin de permettre aux visiteurs d'un jour de découvrir la vie récurrente du lieu. 
Pour rompre avec le positionnement habituel des jeunes dans les centres de sciences, Inmédiats valorise leur expression et les met en situation de concevoir et de réaliser par eux-mêmes comme ici lors du workshop organisé en présence de César Harada en mars 2015.

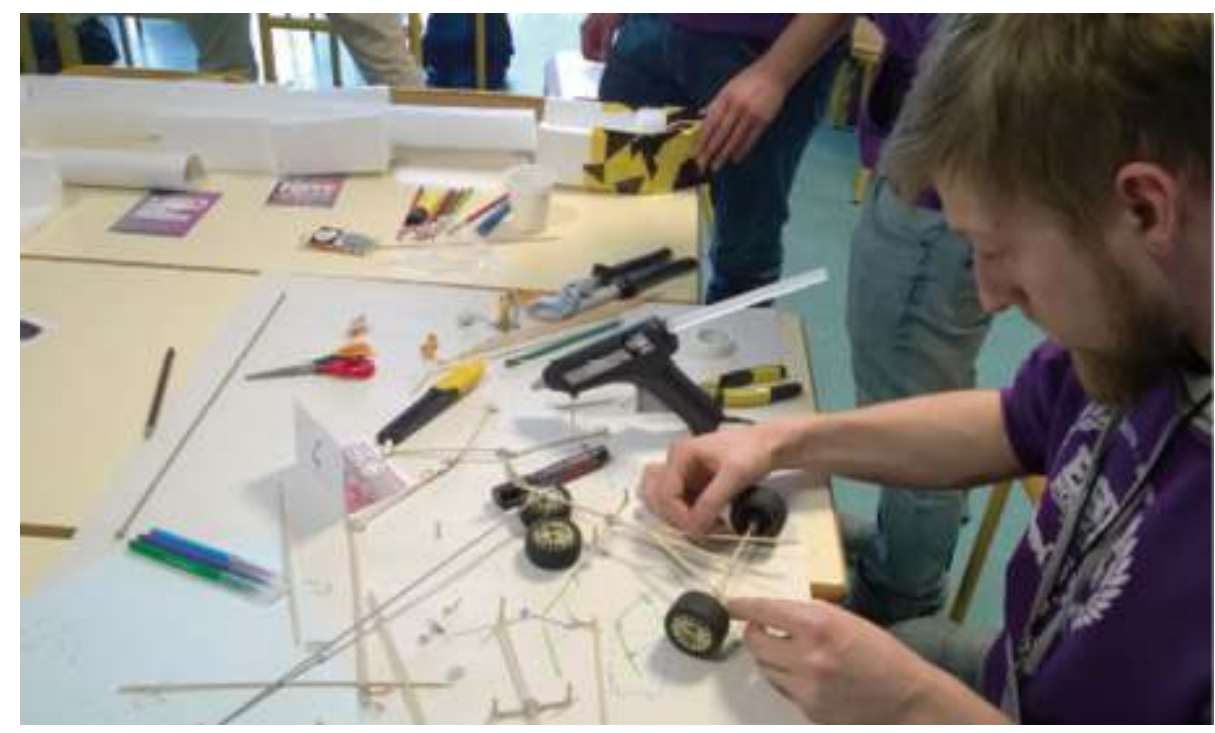

(c) Relais d'sciences/G. Dupuy

Le site devient ainsi un outil idéal pour travailler sur les parcours des compétences des jeunes autour de projets concrets. Il favorise les rapprochements entre ces jeunes et les professionnels du territoire, réinventant ainsi les forums métiers ou les rencontres de recrutement.

\section{Intégrer l'écosystème territorial, spécifiquement celui de l'économie numérique}

Depuis sa fondation en 1998, Relais d'sciences a construit son projet culturel hors les murs, en tissant d'innombrables relations avec les acteurs du territoire. L'étude du Conseil Économique, Social et Environnemental Régional Basse-Normandie réalisée en 2013 a montré la diversité des opérateurs de la culture scientifique et technique en région 5 .

La MRI ajoute à ces opérateurs historiques un lien important avec d'autres acteurs majeurs du territoire comme ceux de la formation professionnelle ou de l'économie numérique. La MRI a ainsi été désignée comme le bâtiment totem de la French Tech à Caen. Elle collabore étroitement avec les agences, dispositifs et réseaux d'innovation et de développement économique.

Les activités qu'elle accueille émanent des acteurs culturels, scientifiques, éducatifs, économiques et artistiques créant ainsi un terrain idéal pour stimuler l\&apos ;imagination, sortir de son cadre habituel et bousculer les idées reçues. Cette mixité favorise voire accélère l'émergence de projets collectifs. 


\section{Assurer la durabilité du projet par un modèle économique moins dépendant des subventions publiques}

30 La restriction des moyens publics (État et collectivités) délivrés par subventions récurrentes impose de repenser en profondeur les modalités de financement de nos centres. La MRI doit donc inventer un nouveau modèle pour assurer son développement économique. Son fonctionnement courant est estimé à environ un million d'euros par an avec une douzaine d'emplois. Plusieurs pistes sont explorées simultanément à cette fin :

31 - La MRI est la propriété de Relais d'sciences en indivision avec la Chambre de Commerce et d'Industrie Caen-Normandie qui en détient $14 \%$. Les équipements sont la propriété intégrale de Relais d'sciences. Cet actif facilite la mobilisation d'une ressource par la location d'espaces évènementiels et de travail. La totalité du bâtiment peut ainsi être proposée à la location, avec ses services. Cette propriété permet également à Relais d'sciences de maîtriser le fonctionnement du bâtiment en optimisant sa gestion et son exploitation ;

32 - l'écosystème réuni dans la résidence de projets est un atout considérable dans la réponse à des appels d'offres régionaux, nationaux et européens. Il permet une réponse collective très rapide. La MRI apparait d'ores et déjà comme le lieu capable de porter ou de participer à des projets numériques innovants avec le public. Elle apporte une plusvalue dans les dossiers des grands opérateurs en région (collectivités, COMUE...) ;

33 - les dépenses liées à la programmation du lieu sont sérieusement diminuées par le principe de co-programmation décrit ci-dessous. Au prix d'un partage de la gouvernance, il permet la conduite d'opérations à destination du public portées par une masse salariale indépendante de Relais d'sciences. Chaque partie est gagnante : le public bénéficie d'une programmation diversifiée portée par des professionnels, les partenaires ont accès à moindre frais à un lieu visible, reconnu et très bien équipé. Relais d'sciences peut ainsi se concentrer sur la mobilisation et l'articulation des projets plutôt que sur leur conception et leur animation ;

34 - l'investissement privé est l'une des voies explorées. Plusieurs groupes industriels s'intéressent à la démarche originale de la MRI, notamment pour se positionner au regard de l'innovation. Des discussions sont donc en cours pour dynamiser les investissements du lieu, notamment en équipements et machines outils ;

35 - dernières pistes de ressources, les prestations du Living Lab et du Fab Lab, du bar et de la boutique, contribuent à l'équilibre des comptes. Le public a un accès gratuit au site et participe, par ses achats ou inscriptions dans des workshops et formations, au financement de la programmation. 
Le modèle économique de la MRI est en partie basé sur la location des espaces en dehors des temps d'ouverture public.

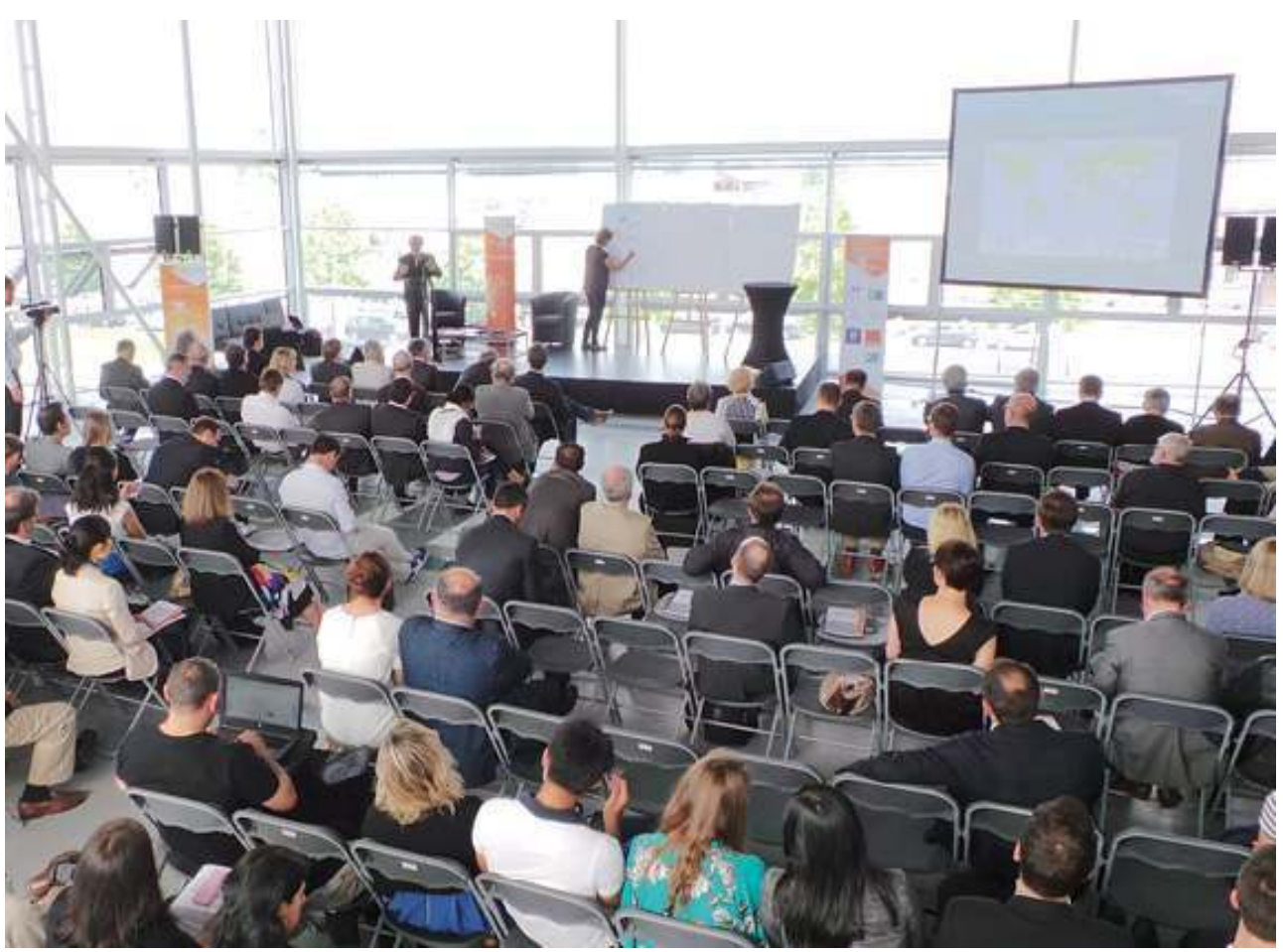

(C) Relais d'sciences/G. Dupuy

\section{Gouvernance et conduite du projet d'investissement}

Le calendrier très serré du programme Inmediats (quatre ans) a incontestablement été un atout majeur pour la construction de la MRI. L'impératif de consommation des subventions allouées dans un temps très restreint a imposé un tour de table financier et un choix des entreprises très rapide, tout en respectant le cadre formel des marchés publics.

Devant les difficultés liées à l'acquisition du terrain via les collectivités partenaires, il est apparu indispensable que Relais d'sciences soit maître d'ouvrage. Cette disposition, non prévue à l'origine, fait aujourd'hui de l'association le propriétaire de la MRI et garantit ainsi une partie importante de son modèle économique à venir.

Un comité de pilotage a permis aux financeurs de rester informés de l'état d'avancement du projet et de participer au choix de la maîtrise d'œuvre qui signe le bâtiment. Relais d'sciences a délégué la maîtrise d'ouvrage à une société spécialisée, Normandie Aménagement, pour s'assurer de la bonne conduite juridique et opérationnelle du chantier. Relais d'sciences a ainsi pu se concentrer, pendant deux ans, sur les préconisations d'usage du bâtiment garantissant les objectifs cités ci-dessus et travailler au développement de son projet culturel, partenarial et économique. Cette maitrise d'ouvrage très présente, tant auprès du programmiste, le Cabinet Aubry \& Guiguet, que de la maîtrise d'œuvre, l'agence Bruther, s'est faite à effectifs constants au sein du CCSTI tout en maintenant son activité culturelle courante hors les murs (6). 
Seule une reconversion complète de Relais d'sciences sur le programme Inmediats permettait de concilier ces objectifs à moyens constants. Le calendrier de construction (un an), le budget (environ six millions pour le foncier, le bâti et les équipements) et le cahier des charges étaient très exigeants. À l'heure de la prise en main de ce bâtiment, et en mettant de côté les aléas habituels dans ce type de projets, le résultat est à la hauteur des attentes.

\section{Découverte du bâtiment}

40 La MRI se présente comme une place publique verticale constituée de trois duplex (R1 à R3) surmontés par un toit terrasse partiellement occupé par un dôme (R4). Un parvis sous bâtiment (R0), d'un volume équivalent, rehausse l'ensemble et offre sa première place couverte à Caen. Les circulations et les locaux techniques se situent en périphérie des grands plateaux dès lors libérés de tout poteau ou couloir inutile. L'ensemble présente un caractère industriel par le traitement brut des bétons, les bardages, les plafonds techniques apparents, les garde-corps et escaliers galvanisés. La présence de coussins gonflables ETFE (7) faisant office de vitrages renforce l'aspect innovant de l'ouvrage.

41 Par son originalité et sa hauteur, la MRI contribue à redessiner l'espace urbain en extrémité de la presqu'île de Caen (600 hectares) dont la rénovation débute tout juste. D'ici 2019, un parc se dressera devant la MRI et établira le lien avec la nouvelle bibliothèque et le centre ville. Le tramway arrivera devant la MRI.

42 Le bâtiment déploie $2095 \mathrm{~m} 2$ utiles, la terrasse en toiture et le parvis ajoutant chacun 400 $\mathrm{m} 2$ à l'ensemble. Chaque duplex, ainsi que le toit, sont utilisables indépendamment les uns des autres. Ces duplex comportent une mezzanine, de taille et de configuration variables, pouvant couvrir jusqu'à la moitié de la surface de l'étage, et offrant des fonctionnalités complémentaires (salles de réunion, espaces de déambulation ou de bureau). Classé Bâtiment ERP (Établissement Recevant du Public) de 3e catégorie, la MRI dispose de cinq classifications ( $L, M, N, R, Y \& T$ ), reflets de la diversité de ses activités. Une visite verticale permet de découvrir ses différentes fonctionnalités. 


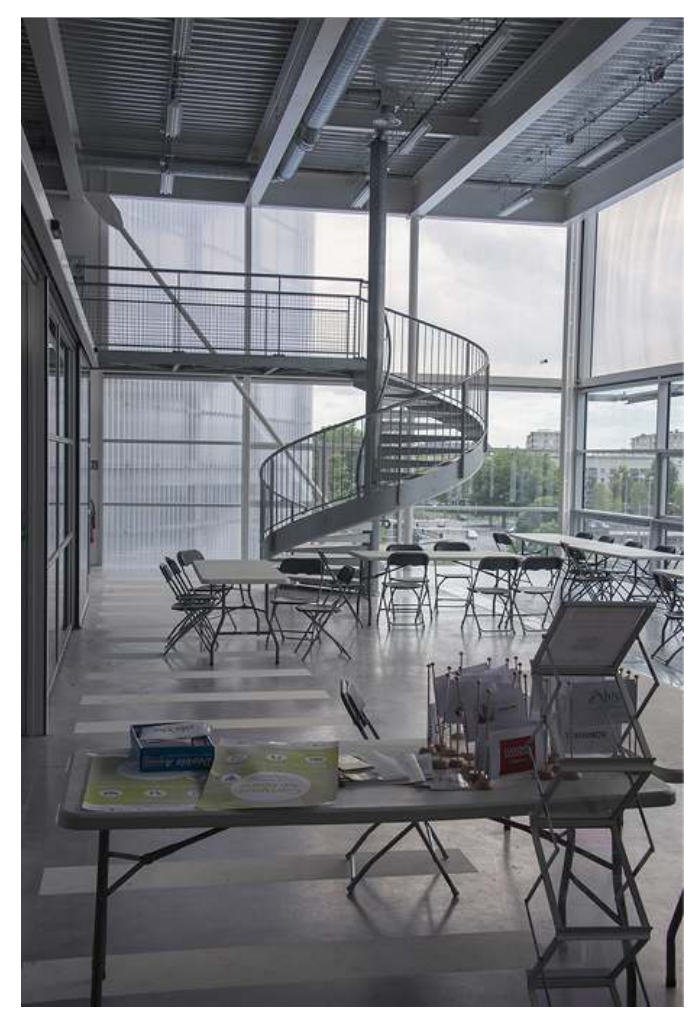

@ Vincent Connétable

$$
\text { À }
$$

À l'exception du 3e étage qui accueille la résidence de projets et nécessite une organisation avec des partenaires conventionnés, la totalité du bâtiment est en libre accès 
pour les publics. L'absence de billetterie et de bornes d'accueil traditionnelles renverse la relation au site. Celui-ci devient un lieu fréquentable au quotidien, proposant des espaces interstitiels, un bar et un toit terrasse invitant à s'approprier librement l'espace.

Pour répondre aux attentes croissantes en matière d'usages numériques, le bâtiment dispose d'atouts technologiques discriminants : une couverture wifi complète en haute densité (200 usagers connectables simultanément), une liaison Internet en fibre optique sur tout le bâtiment (parvis et toit compris), un système éclairage, son et audiovisuel de grande qualité permettant notamment la gestion simultanée des évènements audiovisuels entre les étages ou en streaming.

\section{Co-construction de la programmation culturelle}

50 Au-delà de son aspect et de ses fonctionnalités, la MRI se distingue par une particularité majeure. Pour être en phase avec sa volonté de créer un lieu favorisant l'innovation ouverte, l'équipe de Relais d'sciences a choisi de partager sa gouvernance programmatique.

51 Depuis plusieurs mois, tous les lundis midi, les futurs résidents de la MRI se réunissent autour d'un sandwich pour inventer collectivement la gouvernance et le fonctionnement de la résidence de projets. Inspiré des principes du That Camp ${ }^{6}$, cette démarche fait appel à l'intelligence collective pour régler des questions que l'équipe de Relais d'sciences n'a pas le temps d'approfondir ou pour lesquelles elle ne dispose pas du recul ou de l'expertise nécessaire.

52 Initiées avec les partenaires proches du projet, ces réunions se tiennent désormais avec un cercle élargi. Tous les membres de ce groupe sont issus de structures désirant intégrer la résidence de projets de la MRI : entreprises, associations, chercheurs, membres de collectivités ou de réseaux consulaires, artistes et enseignants.

53 Deux sujets sont choisis chaque semaine parmi une vingtaine proposée par Relais d'sciences autour de quatre thèmes :

54 - le cadre administratif de travail : conventions, assurances, contrôle d'accès...;

55 - apprendre à vivre ensemble : objectifs et valeurs de la MRI, respect des autres dans l'espace de travail (bruit, nettoyage, cuisine...), acculturation mutuelle aux méthodes de travail...;

56 - organiser et faciliter la programmation: choix programmatiques, modes de participation, mobilisation des espaces, moyens techniques et humains, co-construction des formats de médiation, communication interne et externe ;

57 - fonctionnement quotidien : horaires de travail, installation des mobiliers, mobilité dans le bâtiment, stockage, ménage... 
L'activité du Fab Lab, tout comme celle du Living Lab, est prototypée depuis deux ans hors les murs.

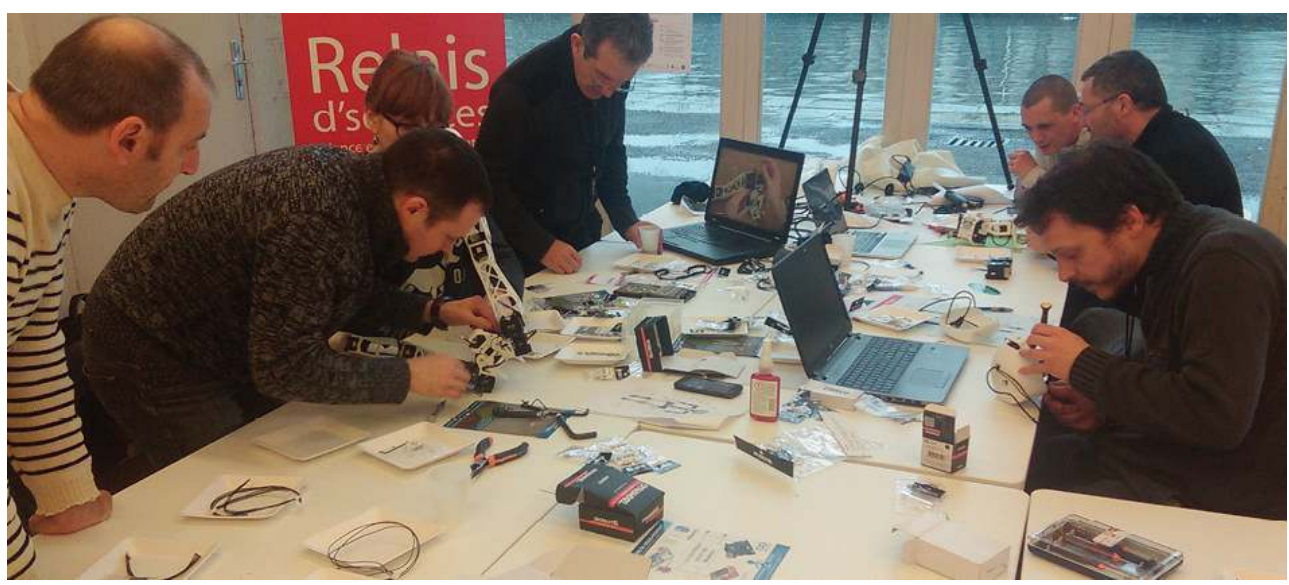

(C) Relais d'sciences/G. Dupuy

Le résultat des discussions est mis en ligne en temps réel pour un partage avec les membres absents. L'objectif est de mettre en œuvre toutes ces résolutions partant du principe qu'elles fédèrent les attentes, motivations et engagements des partenaires impliqués dans la démarche. À terme, une centaine de personnes est amenée à intégrer cette résidence de projets aux côtés de l'équipe de Relais d'sciences.

La programmation se construit en 2015 pour être présentée dans sa forme définitive à partir de 2016 ${ }^{7}$. Les actions de Living Lab et l'activité de Fab Lab sont prototypées depuis deux ans hors les murs pour se déployer rapidement dans la MRI et fonder le cœur de la proposition aux publics. Elles donnent le fil conducteur des ateliers, installations et rencontres organisées à la MRI.

Créer la MRI est devenu un projet de médiation en soi, les usages des communautés de publics étant au cœur des réflexions de l'équipe de Relais d'sciences et de ses partenaires pour concevoir chaque espace, chaque fonction du bâtiment. L'objet est, in fine, particulièrement insolite dans son architecture, sa classification, sa programmation, son économie. Il constitue un tiers-lieu qui place l'innovation et l'imagination au cœur de sa proposition culturelle et de sa relation au territoire.

\section{NOTES}

1. Pour tout connaître du programme Inmediats et accéder à ses nombreux documents téléchargeables, voir www.inmediats.fr. Inmediats regroupe six centres de sciences français : Cap sciences à Bordeaux, Science Animation à Toulouse, La Casemate à Grenoble, Universcience à Paris, Relais d'sciences à Caen et l'Espace des sciences à Rennes.

2. Responsible Research and Innovation : la notion de R.R.I., élaborée par l'Union européenne en novembre 2014, implique l'intégration et l'engagement des acteurs sociétaux dans les processus 
de recherche et de développement, grâce notamment à des approches participatives et inclusives.

3. Voir Livret Inmediats Living Lab sur www.inmediats.fr/Retour d'expérience

4. Picq, P. Un paléoanthropologue dans l'entreprise. S'adapter et innover pour survivre. Eyrolles, 2012, $256 \mathrm{p}$.

5. Savournin, H. et Hugo, P. La culture scientifique et technique en Basse-Normandie : vers une nouvelle ambition. CESER de Basse-Normandie, octobre 2012.

6. Le That Camp est une réunion ouverte, peu coûteuse dans son organisation, invitant des partenaires choisis pour leur diversité de métiers ou de point de vue à construire ensemble des solutions à des questions proposées sur place.

7. ) Le 16 décembre 2015, la MRI accueillera la 4e édition des ateliers Inmediats. Ce rendez-vous, ouvert aux professionnels de la culture scientifique et technique, est l'occasion de partager les réalisations nées d'Inmediats. Cette année, le fil rouge est la création des nouveaux espaces culturels autour de la recherche et de l'innovation.

\section{RÉSUMÉS}

Singulière par son architecture, sa classification, sa programmation et son économie, la Maison de la Recherche et de l'Imagination a été conçue comme un nouvel outil de partage de la connaissance et de culture de l'innovation : son directeur expose ici les principes qui ont guidé sa construction et la démarche qui sous-tend son fonctionnement.

\section{INDEX}

Mots-clés : Maison de la recherche et de l'innovation, Relais d'sciences, innovation, CCSTI

\section{AUTEUR}

BRUNO DOSSEUR

directeur de Relais d'sciences et de la Maison de la Recherche et de l'Imagination à Caen bdosseur@relais-sciences.org 